\title{
Distribuição da gordura corporal e componentes da prática de ativida- des físicas no lazer em mulheres pós- climatério: estudo transversal com usuárias do Sistema Único de Saúde
}

\section{Body fat distribution and components of leisure-time physical activity in post-menopausal women: cross-sectional study among users of Brazilian Public Healthcare System}

Bruna Locci', Bruna C. Turi², Lia G. M. Araújo², Rômulo A. Fernandes ${ }^{3}$, Jamile S. Codogno ${ }^{3}$, Henrique L. Monteiro ${ }^{4}$

\begin{abstract}
RESUMO
Modelo do estudo: Estudo transversal. Objetivo: Verificar o impacto da prática de atividades físicas no lazer sobre a distribuição de massa gorda de mulheres pós-climatério, usuárias dos serviços de atenção básica à saúde. Métodos: A amostra foi composta por 176 mulheres na menopausa, com idade $\geq 50$ anos, usuárias de cinco Unidades Básicas de Saúde de Bauru, São Paulo e selecionadas de forma aleatória. A composição corporal foi expressa por quantidade em gramas de gordura total, central e massa magra, obtida por exame de absortometria radiológica de dupla energia (DXA). Para avaliar a prática de atividade física no lazer utilizou-se a segunda seção do questionário de Baecke, considerando intensidade, duração e tempo prévio de engajamento. O nível econômico foi avaliado pelo questionário da Associação Brasileira de Empresas de Pesquisa. Correlação de Spearman e regressão linear indicaram o relacionamento entre indicadores de gordura corporal e componentes da prática de atividades físicas no lazer. Resultados: A maior parte das participantes situou-se na classe média, com idade de $65,9 \pm 8$ anos e o início da menopausa ocorreu aos 47,5 \pm 6 anos. Foi observada relação entre maior intensidade e maior tempo prévio de engajamento nas atividades de lazer com menores valores de gordura na região do tronco, porém, quando corrigidos por alguns fatores de confusão, esta relação deixou de apresentar significância estatística. Conclusões: Para esta população, atividades físicas praticadas no tempo destinado ao lazer ocorrem com intensidade, duração e tempo prévio de engajamento insuficientes para provocar alterações significativas na composição corporal.
\end{abstract}

Palavras-chave: Obesidade; Atividade Motora; Menopausa.

1. Mestranda no Programa de Pós Graduação em Ciências da Motricidade - Universidade Estadual Paulista "Júlio de Mesquita Filho", Campus de Rio Claro.

2. Doutoranda no Programa de Pós Graduação em Ciências da Motricidade - Universidade Estadual Paulista "Júlio de Mesquita Filho", Campus de Rio Claro.

3. Professor Assistente Doutor do Departamento de Educação Física - Universidade Estadual Paulista "Júlio de Mesquita Filho", Campus de Presidente Prudente.

4. Professor Assistente Doutor do Departamento de Educação Física - Universidade Estadual Paulista "Júlio de Mesquita Filho", Campus de Bauru.
Correspondência: Universidade Estadual Paulista. Faculdade de Ciências. Departamento de Educação Física. Av. Eng. Luiz Edmundo Carrijo Coube, 14-01 CEP: 17033-360 - Bauru/SP. E-mail: brunalocci@gmail.com

Artigo recebido em 09/04/2014 Aprovado para publicação em 25/08/2014 


\begin{abstract}
Study design: Cross-sectional study. Aim: To investigate whether leisure-time physical activity can impact on fat mass distribution in post-menopausal women, users of Brazilian Public Healthcare System. Methods: The sample was composed by 176 postmenopausal women aged $\geq 50$ years. Body composition was expressed as quantity in grams of total and central fat mass and lean mass, obtained by dual Xray absorptiometry (DXA). To assess leisure-time physical activity we used the second section of Baecke's questionnaire considering intensity, duration and prior engagement in physical activity practice. Economic status was assessed by a specific and previously validated Brazilian questionnaire. Spearman correlation and linear regression assessed the relationship between indicators of body fat and components of leisure-time physical activity. Results: Most part of participants was middle-income, with mean age of 65,9 \pm 8 years and the onset of menopause occurred at $47,5 \pm 6$ years. We observed relationship between higher intensity and prior engagement in leisure-time physical activities with lower levels of central fat, however, when adjusted for some confounding factors, such as this relationship is no longer statistical significant. Conclusion: For this population, intensity, duration and prior engagement in leisure-time physical activity are insufficient to cause significant changes in body composition.
\end{abstract}

Key-words: Obesity; Motor Activity; Menopause.

\section{Introdução}

A obesidade atualmente é considerada doença crônica de abrangência mundial. ${ }^{1}$ Com a globalização, a silenciosa pandemia rapidamente se alastrou também pelos países em desenvolvimento, fazendo com que estes muitas vezes enfrentem duplo fardo - a carga de doenças infecciosas que acompanham a desnutrição e a crescente onda de doenças crônicas associadas à obesidade e estilos de vida ocidentais. ${ }^{2}$

Com taxas que quase dobraram desde 1980, estimativas atuais são de 1,5 bilhões de adultos com sobrepeso ou obesidade e, para 2030, existe a projeção de que mais de um bilhão de adultos se tornem obesos. Esta situação é alarmante, na medida em que, juntamente com o excesso de peso, aumentam significativamente inúmeros outros problemas de saúde com forte impacto sobre a produtividade da população., $3,4,5$

Devido aos enormes custos, tanto públicos quanto pessoais, e a dificuldade em se perder peso uma vez obeso, a prevenção é fundamental. Retardar o aumento dessa epidemia em grande escala requer esforços multifacetados, dentro de cada país e em todo o mundo, para melhorar, entre outras medidas de saúde pública, as escolhas alimentares e os níveis de atividade física das pessoas. ${ }^{6,7}$

Pesquisas recentes têm apontado para a importância do aprofundamento no estudo da obesidade, porque o excesso de gordura corporal, principalmente a abdominal, está diretamente relacionado às alterações do perfil lipídico, aumento da pressão arterial, hiperinsulinemia, entre outras complicações à saúde. ${ }^{8,9,10}$
Entretanto, ao se buscar verificar a relação entre obesidade, distribuição de gordura corporal e prática de atividades físicas, inúmeros estudos utilizam apenas o domínio do lazer como parâmetro de avaliação, em grande parte, devido à recomendações como a do Physical Activity Guidelines for Americans $^{11}$, considerando as evidências científicas de que a prática de atividades físicas nesse domínio é a que parece ter maior impacto sobre o controle e prevenção de doenças crônicas não transmissíveis. ${ }^{12-15}$

Acerca desta questão, Buonani et al. ${ }^{16}$, analisando a relação entre a prática de atividade física e composição corporal, observaram que mulheres que acumularam $\geq 150$ minutos semanais de atividades físicas em intensidade moderada/vigorosa apresentaram valores inferiores de massa gorda total, quando comparadas àquelas que não atingiam esta meta. Entretanto, apesar dos resultados benéficos à saúde das mulheres analisadas, a investigação em questão não descreveu detalhes sobre a condição sócio econômica das participantes e, tampouco se eram usuárias do sistema público de saúde, o que deixa em aberto uma lacuna quanto a especificidade da população estudada.

Neste contexto, pesquisadores da área de saúde têm envidado esforços para averiguar se a adoção de hábitos saudáveis, como a prática regular de atividades físicas, pode contribuir para o controle e a prevenção de doenças crônicas não transmissíveis em populações mais específicas. Dessa maneira, o objetivo do presente estudo foi verificar se a prática de atividades físicas no lazer pode impactar sobre a distribuição de massa gorda de mulheres pós-climatério 
e de classe social média, usuárias dos serviços de atenção básica à saúde.

\section{Material e Métodos}

\section{População e amostra}

O desenho amostral foi calculado com base na população de estudo anterior, ${ }^{17,18}$ que considerou que $40 \%$ da população do Estado de São Paulo é usuária de algum plano de saúde, enquanto os demais (60\%) estão cobertos pelo SUS. ${ }^{19}$ Desse modo, considerando o percentual da população atendido exclusivamente pelo SUS, um erro amostral arbitrário de 3,8\% e significância de 5\%, a amostra total a ser avaliada foi estimada em 960 indivíduos, dos quais foram avaliados 963 pacientes em cinco unidades básicas de saúde (UBS) do município de Bauru, São Paulo.

Dos 963 pacientes selecionados aleatoriamente, 707 eram mulheres, às quais foram consideradas como população de estudo para definição do cálculo amostral. O estudo de Saravi e Sayegh ${ }^{20}$, que investigou a composição corporal, osteoporose e atividade física em mulheres pós-menopausa, foi utilizado como base para o cálculo da amostra, que indicou a necessidade de um mínimo de 144 pacientes. Para efeito de estudo, a amostra foi composta por 176 mulheres na menopausa, com idade $\geq 50$ anos e usuárias de cinco Unidades Básicas de Saúde de Bauru/SP, às quais foram selecionadas mediante sorteio.

Todas as pacientes assinaram o termo de consentimento livre e esclarecido e o projeto foi aprovado pelo Conselho de Ética da Secretaria de Saúde de Bauru e pelo Comitê de Ética em Pesquisa (Processo $\left.n^{\circ} .24856 / 13\right)$.

\section{Variáveis de Estudo}

\section{Composição corporal}

A composição corporal, expressa pela quantidade em gramas de gordura total e central, foi obtida por exame de absortometria radiológica de dupla energia (DXA) com scanner de corpo inteiro através do equipamento da marca Hologic ${ }^{\circledR}$, Discovery Wi. Adicionalmente, o índice de massa corporal (IMC), calculado através da utilização dos valores de massa corporal e estatura $\left(\mathrm{kg} / \mathrm{m}^{2}\right)$, apontou presença de sobrepeso para valores $>25 \mathrm{~kg} / \mathrm{m}^{2}$ e obesidade para $>30 \mathrm{~kg} /$ $\mathrm{m}^{2} .{ }^{21}$ A circunferência de cintura (CC) foi realizada e os valores $\geq 0,88$ metro foram considerados como alterados. $^{22}$

\section{Atividade física no lazer}

A prática de atividades físicas no lazer foi avaliada pelo questionário de Baecke et al $^{23}$, o qual utiliza um escore total para atividade física habitual e um escore adimensional para cada um dos três diferentes domínios de atividade física, como segue: ocupacional, esportes e locomoção. A prática atual de exercícios físicos no lazer foi avaliada por meio da segunda sessão do instrumento, referente a atividades esportivas durante horários de lazer, e foram considerados, particularmente: i) quantidade de minutos que praticavam determinados exercícios físicos ou esportes na semana; ii) intensidade na qual eram realizadas tais atividades, e iii) há quanto tempo estavam engajadas nessa prática.

\section{Potenciais fatores de confusão}

Através de entrevista estruturada foram obtidos os seguintes dados: i) etnia, onde a paciente poderia responder entre as alternativas caucasiana, negra, parda e asiática; ii) idade cronológica, confirmada em prontuário clínico; iii) idade em que a paciente entrou na menopausa, informação auto referidas; iv) poder aquisitivo, avaliado por meio de questionário desenvolvido pela Associação Brasileira de Empresas de Pesquisa (ABEP) ${ }^{24}$, no qual a subdivisão se dá de $\mathrm{A}$ (mais alto) até E (mais baixo). Para classificação das pacientes em grupos por poder aquisitivo foi adotado: classes A1, A2, B1 e B2 como classe alta, C1 e C2 como classe média e classes D e E como classe baixa.

\section{Análise estatística}

Para variáveis numéricas, estatística descritiva foi composta por valores de média, desvio padrão (DP) e intervalo de confiança de $95 \%$ (IC95\%). Variáveis categóricas foram expressas em taxas (\%). A correlação de Spearman foi usada para avaliar a relação entre a variável dependente e independente e, modelos de regressão linear ajustados por etnia, idade em que entrou na menopausa e idade cronológica avaliaram o relacionamento entre indicadores de gordura corporal e componentes da prática de atividades físicas no lazer. A significância estatística foi fixada em 5\% e o software estatístico Estat versão 5.0 foi usado para todas as análises.

\section{Resultados}

Conforme indicado anteriormente, 176 mulheres na menopausa completaram todas as avaliações 
previstas (Tabela 1 e Tabela 2). Com relação ao nível econômico, a maior parte das participantes situou-se na classe média $(60 \%, \mathrm{n}=106)$, seguida pela classe alta $(28 \%, n=49)$ e classe baixa $(n=21,12 \%)$. Quanto às variáveis de composição corporal, 19,3\% das mulheres apresentaram IMC dentro das faixas de normalidade $(n=34), 36,9 \%$ foram classificadas como sobrepesadas $(n=65)$ e $43,8 \%$ obesas $(n=77)$. Para circunferência de cintura, 74,4\% apresentaram valo- res alterados $(n=131)$. Exame de absorciometria radiológica de dupla energia indicou que a média de gordura corporal total das avaliadas foi $30,45 \mathrm{Kg}$ e massa magra 40,27 Kg, com razão média entre gordura total e massa magra de 0,75 . Em média, o início da menopausa ocorreu aos 47,5 \pm 6 anos e, esta variável não se relacionou com os indicadores da gordura corporal analisados, bem como, com os indicadores da atividade física no lazer ( $p>0,05)$.

Tabela 1. Valores de média, desvio padrão e respectivos intervalos de confiança das variáveis numéricas consideradas para o estudo (Bauru, $\mathrm{n}=176$ ).

\begin{tabular}{lcc}
\hline Variáveis & Média \pm DP & (IC95\%) \\
\hline Idade (anos) & $65,9 \pm 8,1$ & $(64,4-66,9)$ \\
Idade da menopausa (anos) & $47,5 \pm 5,8$ & $(46,6-48,4)$ \\
Gordura & & \\
$\quad$ Tronco (gramas) & $16.297,92 \pm 5.033,4$ & $(15.519,1-17.016,7)$ \\
$\quad$ Total (gramas) & $30.450,88 \pm 9.049,8$ & $(29.104,5-31.797,1)$ \\
\hline
\end{tabular}

Notas: DP= desvio-padrão; IC95\%= intervalo de confiança de $95 \%$.

Tabela 2. Valores absolutos e relativos das variáveis categóricas consideradas para o estudo (Bauru, n=176).

\begin{tabular}{lcc}
\hline Atividades físicas no lazer & N & $(\%)$ \\
\hline Pratica Esporte & & $(50)$ \\
Sim & 88 & $(50)$ \\
Não & 88 & 0 \\
Intensidade & & $(97,7)$ \\
Baixa & 0 & $(2,3)$ \\
Moderada & 86 & \\
Intensa & 2 & 0 \\
Duração & & $(4,5)$ \\
$<1 \mathrm{~h}$ & 0 & $(4,5)$ \\
$1-2 \mathrm{~h}$ & 04 & $(25)$ \\
$2-3 \mathrm{~h}$ & 04 & $(65,9)$ \\
$3-4 \mathrm{~h}$ & 22 & \\
$>4 \mathrm{~h}$ & 58 & 0 \\
Engajamento Prévio & \multicolumn{2}{c}{} \\
$<1$ mês & 0 & $(2,27)$ \\
$1-3$ meses & 02 & $(2,27)$ \\
$4-6$ meses & 02 & $(3,41)$ \\
$7-9$ meses & 03 & $(92)$ \\
$>9$ meses & 81 &
\end{tabular}

Notas: $\mathrm{N}=$ número amostral; $(\%)=$ porcentagem; $\mathrm{h}=$ horas. 
Por outro lado, a variável idade $(65,9 \pm 8,1$ anos) apresentou associação significante com a gordura corporal total $(\mathrm{rho}=-0.30 ; \mathrm{p}$-valor $=0,001)$ e a localizada na região do tronco $(\mathrm{rho}=-0.28$; $\mathrm{p}$-valor $=$ 0,001). Entretanto, não se relacionou com os componentes da atividade física no lazer, tais como intensidade, duração e tempo de engajamento na prática da atividade física ( $>>0,05)$.

No que se refere à relação entre prática de atividades físicas no lazer e as variáveis relativas à distribuição da gordura corporal, não foram encontradas diferenças significativa para a gordura total (Tabela 3). Por outro lado, a intensidade ( $r h o=-0.17$; p-valor $=0,022)$ e o tempo prévio de engajamento $(\mathrm{rho}=$ 0.15 ; $\mathrm{p}$-valor $=0,041$ ) nas atividades de lazer relacionaram-se com menores valores de gordura na região do tronco. No entanto, quando os dados sobre o nível de atividade física foram ajustados por grupo étnico e idade de entrada na menopausa, apenas a intensidade apresentou diferença estatística significante para a gordura do tronco. Portanto, no modelo final, no qual foi realizado ajuste por idade cronológica, esta relação deixou de expressar significância estatística.

\section{Discussão}

O presente estudo buscou verificar como componentes da prática de atividades físicas no domínio lazer impactam na distribuição de massa gorda de mulheres pós menopausa, de classe média e usuárias dos serviços de atenção básica à saúde. Os resultados indicaram que a intensidade e o tempo prévio de engajamento nas atividades de lazer relacionaram-se com menores valores de gordura na região do tronco, porém, quando corrigidos por alguns fatores de confusão, esta relação deixou de apresentar significância estatística.

No que se refere à caracterização da amostra, a maioria das participantes era oriunda de classes sociais média e baixa, indicando tratar-se de população de baixa renda. ${ }^{25}$ Em relação à composição corporal, $80,7 \%$ das mulheres foram classificadas como sobrepesadas ou obesas. Este fato pode ser explicado, em parte, pelas mudanças na composição corporal que ocorrem com o avanço da idade, quando é esperado aumento das concentrações de gordura corpórea e diminuição de massa magra. ${ }^{26}$

A menopausa é caracterizada pela interrupção do ciclo menstrual e diminuição da produção de estrogênio, resultando em mudanças significativas na composição corporal, como aumento do peso e da adiposidade, principalmente na região do tronco ${ }^{27}$. Entretanto, nossos resultados não apontaram relação entre a idade média de entrada na menopausa com os indicadores de gordura corporal analisados, bem como, esta também não se relacionou com componentes da atividade física no lazer. Uma explicação plausível para este fato pode ser a utilização somente

Tabela 3: Relacionamento entre indicadores de gordura corporal e componentes da prática de atividades físicas no lazer em mulheres na menopausa (Bauru, $\mathrm{n}=176$ ).

\begin{tabular}{|c|c|c|c|c|}
\hline \multirow[b]{2}{*}{ Variável independente } & \multirow{2}{*}{$\begin{array}{l}\text { Correlação de } \\
\text { Spearman } \\
\text { rho }\end{array}$} & \multirow[b]{2}{*}{$p$-valor } & \multicolumn{2}{|c|}{ Regressão Linear } \\
\hline & & & Modelo 1 & Modelo 2 \\
\hline \multicolumn{5}{|l|}{ Gordura total $(\mathrm{g})$} \\
\hline Intensidade & -0.12 & 0.105 & - & - \\
\hline Duração/semanal & -0.07 & 0.344 & 一 & -- \\
\hline Tempo prévio & -0.10 & 0.158 & - & 一 \\
\hline \multicolumn{5}{|l|}{ Gordura de tronco $(\mathrm{g})$} \\
\hline Intensidade & -0.17 & 0.022 & $-759.1(-1495 ;-22.6)$ & $-654.2(-1369 ; 60.6)$ \\
\hline Duração/semanal & -0.11 & 0.130 & 一- & 一- \\
\hline Tempo prévio & -0.15 & 0.041 & $-296.1(-602.2 ; 9.86)$ & $-250.2(-547.3 ; 46.9)$ \\
\hline
\end{tabular}

Notas: rho= correlação de Spearman; Modelo-1 ajustado por etnia e idade em que entrou na menopausa; Modelo-2 ajustado por etnia, idade em que entrou na menopausa e idade cronológica. 
da idade de entrada na menopausa, considerando que os efeitos fisiológicos causados pelo fim da função ovariana, como declínio físico e $\operatorname{cognitivo}{ }^{28}$, são relacionados ao período de tempo em que a mulher se encontra na menopausa. ${ }^{29}$

Em se tratando, especificamente, da idade cronológica, foi encontrada relação significativa com gordura corporal total e localizada na região do tronco, ou seja, quanto mais idosas eram as mulheres da amostra, menores foram os valores de gordura nestas regiões. Estes dados concordam com estudo transversal de Wildman et al. ${ }^{30}$, quando observaram que mulheres com excesso de peso/obesidade eram mais jovens, se comparadas a aquelas que apresentavam padrão eutrófico. Sendo assim, a massa gorda, visceral e subcutânea, tem um aumento na fase adulta, porém, tende a diminuir em idades mais avançadas. ${ }^{31}$

Adicionalmente, em nosso estudo, a idade cronológica não se relacionou com intensidade, duração e tempo de engajamento na prática da atividade física no lazer. Neste caso, a disposição das mulheres para realização destas atividades pode ser reduzida por vários fatores, tais como o estado depressivo na menopausa, que pode comprometer as capacidades físicas e atenuar a relação entre as funções físicas e $\operatorname{cognitivas}^{29}$, barreiras pessoais, como a falta de tempo, dinheiro e cansaço ${ }^{32}$, ou acometimento por alguma doença que limite ou impeça a prática de atividades físicas.

No que se refere à atividade física praticada no lazer e indicadores da gordura corporal, não foi encontrada relação significativa com gordura total. Em contrapartida, Hansen et al..$^{33}$, observaram que mulheres pós-menopausa e fisicamente ativas apresentaram menores valores de massa gorda e maiores de massa magra, quando comparadas a outras com baixo nível de atividades físicas. Neste caso, a falta de associação pode ser, em parte, explicada pela restrição ao domínio lazer na avaliação da prática de atividades físicas, já que, além de atividades físicas praticadas nesse domínio, essas mulheres também precisam trabalhar, se preocupar com afazeres domésticos e se deslocar, muitas vezes a pé, de um lugar para o outro $^{34}$, fazendo com que a recomendação dos 150 minutos semanais do Physical Activity Guidelines for Americans ${ }^{11}$, talvez seja insuficiente para provocar mudanças nos valores de gordura total nessa população específica, devido tanto ao estrato social em que estas mulheres estão inseridas, quanto ao fato de realizarem atividades ocupacionais e de locomoção com intensidades iguais ou mais elevadas que aquelas que realizam 150 minutos de atividade moderada apenas no domínio lazer.

Além disso, para a realidade brasileira, a prática de atividades físicas no domínio lazer não é tão comum entre mulheres quando comparadas aos homens $^{35,36}$, e esse quadro se agrava pelo fato de que mulheres de condições sócio econômicas mais baixas tendem a assumir postos de trabalho menos qualificados e mais exigentes fisicamente ${ }^{37}$, o que reduz a disposição para o engajamento em prática de atividades físicas em outros domínios.

Por outro lado, nossos resultados mostraram que a intensidade e o tempo prévio de engajamento nas atividades de lazer relacionaram-se com menores valores de gordura na região do tronco, mesmo quando os resultados são corrigidos por etnia e idade de entrada na menopausa. Ekelund et $\mathrm{al}^{38}$, apontam que níveis mais elevados de atividades físicas foram significativamente e inversamente associados com mudanças na circunferência da cintura em mulheres pósmenopausa. Estes resultados sugerem que há um efeito da atividade física de nível mais elevado sobre a adiposidade central, independente de uma alteração no peso corporal, considerando uma estratégia útil para a prevenção de doenças crônicas.

Por fim, no modelo final, no qual foi efetuado ajuste por idade cronológica, não foi observada interação entre os mesmos componentes de atividade física de lazer e indicadores da gordura corporal. Dentre os fatores que podem ter contribuído para estes resultados está mais uma vez o viés de classe social, que corrobora com achados de Duca et $a l^{39}$, os quais observaram que a prevalência de inatividade física no lazer, de acordo com o nível sócio econômico, é linearmente decrescente das classes sociais mais altas para as mais baixas. A presente investigação evidencia para a população em questão que o uso da determinação dos níveis de atividade física apenas no lazer pode induzir o pesquisador a um viés de interpretação dos seus achados, face ao qual, recomenda-se sempre considerar o tipo e/ou intensidade das atividades físicas dos outros domínios para efeito de classificação dos sujeitos por níveis de atividades físicas. ${ }^{29}$

Dessa maneira, vale destacar que, talvez, grupos populacionais específicos, oriundos de países em desenvolvimento, não respondam da mesma forma às recomendações de prática de atividades físicas expressas em documentos do Physical Activity Guidelines for Americans ${ }^{11}$, American College of Sports 
Medicine ${ }^{40}$ e outros órgãos especializados na área, indicando que, para o público avaliado, ou seja, mulheres pós menopausa de renda média, a exposição aos 150 minutos de atividade física por semana, praticados em intensidade moderada, a ser realizada durante o período de lazer, não seja suficiente para promover as alterações esperadas sobre a composição corporal, mais especificamente sobre a distribuição de gordura central e total. Assim, estratégias de saúde pública deveriam também considerar subgrupos específicos quando quiserem tratar do aumento da prática de atividades físicas em diferentes domínios, visto que o padrão de comportamento nessa área tem sofrido constantes mudanças nas últimas décadas ${ }^{34}$, o que tem estreita relação tanto com o aumento das taxas de obesidade.

Como limitações do estudo destacam-se: o delineamento transversal, que não permite estabelecer relações de causa-efeito, a falta de informações sobre a utilização de terapia de reposição hormonal, que poderia explicar, em parte, alguns resultados encontrados, e o uso de questionário para identificar os níveis de atividades físicas, já que a população pode reportar montantes superiores aos reais devido a uma maior conscientização da importância da atividade física.

\section{Conclusão}

Conclui-se que atividade física praticada no domínio do lazer ocorre com intensidade, duração e tempo prévio de engajamento insuficientes para provocar alterações significativas na composição corporal de mulheres pós climatério, de classe média e usuárias do sistema público de saúde brasileiro.

\section{Agradecimentos}

Coordenação de Aperfeiçoamento de Pessoal de Nível Superior (CAPES) e Conselho Nacional de Desenvolvimento Científico e Tecnológico (CNPq).

\section{Referências}

1. Malik VS, Willett WC, Hu FB. Global obesity: trends, risk factors and policy implications. Nat Rev Endocrinol. 2013; 9:13-27.

2. Popkin BM, Adair LS, Ng SW. Global nutrition transition and the pandemic of obesity in developing countries. Nutr Rev. 2012; 70:3-21

3. Finucane MM, Stevens GA, Cowan MJ, Danaei G, Lin JK, Paciorek CJ, et al. National, regional, and global trends in bodymass index since 1980: systematic analysis of health examination surveys and epidemiological studies with 960 country-years and 9.1 million participants. Lancet. 2011; 377(9765):557-67.
4. International Obesity Task Force. The Global Obesity Epidemic. 2010. Accessed February 20, 2014.

5. Kelly T, Yang W, Chen CS, Reynolds K, He J. Global burden of obesity in 2005 and projections to 2030. Int J Obes. 2008; 32:1431-7.

6. Apovian $\mathrm{CM}$. The clinical and economic consequences of obesity. Am J Manag Care. 2013; 19(11 Suppl):s219-28.

7. Ladabaum U, Mannalithara A, Myer PA, Singh G. Obesity, abdominal obesity, physical activity, and caloric intake in U.S. adults: 1988-2010. Am J Med. 2014; 127: 717-27.

8. Després JP, Lemieux I. Abdominal obesity and metabolic syndrome. Nature. 2006; 444(7121):881-7.

9. Carr DB, Utzschneider KM, Hull RL, Kodama K, Retzlaff BM, Brunzell JD, et al. Intra-abdominal fat is a major determinant of the national cholesterol education program adult treatment panel III criteria for the metabolic syndrome. Diabetes. 2004; 53:2087-94.

10. Coutinho T, Goel K, Corrêa de Sá D, Carter RE, Hodge DO, Kragelund $\mathrm{C}$, et al. Combining body mass index with measures of central obesity in the assessment of mortality in subjects with coronary disease: role of "normal weight central obesity". J Am Coll Cardiol. 2013; 61:553-60.

11. U.S. Department of Health and Human Services. Physical Activity Guidelines for Americans: be active, healthy, and happy! Washington 2008.

12. Seo DC, Li K. Leisure-time physical activity dose-response effects on obesity among US adults: results from the 19992006 National Health and Nutrition Examination Survey. J Epidemiol Community Health. 2010; 64:426-31.

13. Kwon S, Wang M, Hawkins M. Association between self-reported physical activity and obesity among White, Black, Hispanic, and Asian Americans: 2007 and 2009 brfss. Ethn Dis. 2013; 23:129-35.

14. Mouchacca J, Abbott GR, Ball K. Associations between psychological stress, eating, physical activity, sedentary behaviours and body weight among women: a longitudinal study. BMC Public Health. 2013; 13:828.

15. Shiroma EJ, Sesso HD, Lee IM. Physical activity and weight gain prevention in older men. Int J Obes.2012; 36:1165-9.

16. Buonani C, Rosa CSC, Diniz TA, Christofaro DGD, Monteiro $\mathrm{HL}$, Rossi FE, et al. Prática de atividade física e composição corporal de mulheres na menopausa. Rev Bras Ginecol Obstet. 2013; 35:153-8.

17. Turi BC, Codogno JS, Fernandes RA, Monteiro HL. Associação entre doenças crônicas em adultos e redução dos níveis de atividade física. Medicina (Ribeirão Preto). 2011; 44: 38995.

18. Codogno JS, Fernandes RA, Monteiro HL. Prática de atividades físicas e custo do tratamento ambulatorial de diabéticos tipo 2 atendidos em unidade básica de saúde. Arq. bras. endocrinol. metabol. 2012; 56:6-11.

19. Kilsztajn S, Silva DF, Camara MB, Ferreira VS. Grau de cobertura dos planos de saúde e distribuição regional do gasto público em saúde. Saúde Soc. 2001; 10:35-46.

20. Saravi FD, Sayegh F. Bone mineral density and body composition of adult premenopausal women with three levels of physical activity. J Osteoporos 2013; 2013:1-7.

21. (OMS) ORGANIZAÇÃO MUNDIAL DE SAÚDE. Obesity, Preventing and Managing the Global Epidemic: Report of the WHO Consultation on Obesity. (WHO Technical Report Series 894). World Health Organization: Geneva, 1998. Disponível em: http://www.who.int/nutrition/publications/ obesity_executive_summary.pdf Acesso em: 20 de Jan. 2014. 
22. Peixoto MRG, Benício MHD, Latorre MRDO, Jardim PCBV Circunferência da cintura e índice de massa corporal como preditores da hipertensão. Arq Bras Cardiol. 2006;87:462-70.

23. Baecke JAH, Burema J, Frijters JER. A short questionnaire for the measurement of habitual physical activity in epidemiological studies. Am J Clin Nutr. 1982; 36: 936-42.

24. Associação Brasileira de Empresas de Pesquisa. Dados com base no Levantamento Sócio Econômico 2008 - IBOPE, Disponível em: www.abep.org. 2010.

25. Pinheiro MM, dos Reis Neto ET, Machado FS, Omura F, Yang JH, Szejnfeld J, Szejnfeld VL. Risk factors for osteoporotic fractures and low bone density in pre and postmenopausal women. Rev Saude Publica. 2010;44:479-85.

26. Fielding RA, vellas B, evans WJ, bhasin S, morley je et al. Sarcopenia: an undiagnosed condition in older adults. Current consensus definition: prevalence, etiology, and consequences. International working group on sarcopenia. J Am Med Dir Assoc. 2011;12:249-56.

27. Donato GB, Fuchs SC, Oppermann K, Bastos C, Spritzer PM. Association between menopause status and central adiposity measured at different cutoffs of waist circumference and waist-to-hip ratio. Menopause. 2006; 13:280-5.

28. Sowers M, Pope S, Welch G, Sternfeld B, Albrecht G. The association of menopause and physical functioning in women at midlife. J Am Geriatr Soc. 2001; 49: 1485-92.

29. Ford K, Sowers M, Seeman TE, Greendale GA, Sternfeld B, Everson-rose SA. Cognitive functioning is related to physical functioning in a longitudinal study of women at midlife. Gerontology. 2010; 56:250-8.

30. Wildman RP, Kaplan R, Manson JE, Rajkovic A, Connelly SA, Mackey $\mathrm{RH}$, et al. Body Size Phenotypes and Inflammation in the Women's Health Initiative Observational Study. Obesity. 2011; 19:1482-91.

31. Buffa R, Floris GU, Putzu PF, Marini E. Body composition variations in ageing. Coll Antropol 2011;35:259-65.

32. Reichert FF, Barros AJ, Domingues MR, Hallal PC. The role of perceived personal barriers to engagement in leisure-time physical activity. Am J Public Health. 2007;97:515-9.
33. Hansen RD, Allen BJ. Habitual physical activity, anabolic hormones, and potassium content of fat-free mass in postmenopausal women. Am J Clin Nutr. 2002; 75:314-20.

34. Hallal PC, Victora CG, Wells JCK, Lima RC. Physical inactivity: prevalence and associated va-riables in Brazilian adults. Med Sci Sports Exerc. 2003; 35:1894-900.

35. Berrigan D, Troiano RP, McNeel T, DiSogra C, Ballard-Barbash $R$. Active transportation increases adherence to activity recommendations. Am J Prev Med. 2006; 31:210-16.

36. Nang EEK, Khoo EY, Salim A, Tai ES, Lee J, Van Dam RM. Patterns of physical activity in different domains and implications for intervention in a multi-ethnic Asian population: a cross- sectional study. BMC Public Health. 2010;10:644.

37. Del Duca GF, Nahas MV, de Sousa TF, Mota J, Hallal PC, Peres KG. Clustering of physical inactivity in leisure, work, commuting and household domains among Brazilian adults. Public Health. 2013;127:530-7.

38. Ekelund U, Besson H, Luan J, May AM, Sharp SJ, Brage S, et al. Physical activity and gain in abdominal adiposity and body weight: prospective cohort study in 288,498 men and women. Am J Clin Nutr. 2011;93:826-35.

39. Duca GFD, Rombaldi AJ, Knuth AG, Azevedo MR, Nahas MV, Hallal PC. Associação entre nível econômico e inatividade física em diferentes domínios. Rev Bras Ativ Fís Saúde.2009; 14: 123-31.

40. Haskell WL, Lee IM, Pate RR, Powell KE, Blair SN, Franklin $B A$, et al. Physical activity and public health: updated recommendation for adults from the American College of Sports Medicine and the American Heart Association. Med Sci Sports Exerc. 2007; 39:1423-34.

41. Hallal PC, Knuth AG, Reis RS, Rombaldi AJ, Malta DC, Iser $\mathrm{BPM}$, et al. Tendências temporais de atividade física no Brasil (2006-2009). Rev Bras Epidemiol 2011; 14(1) Supl.: 5360. 